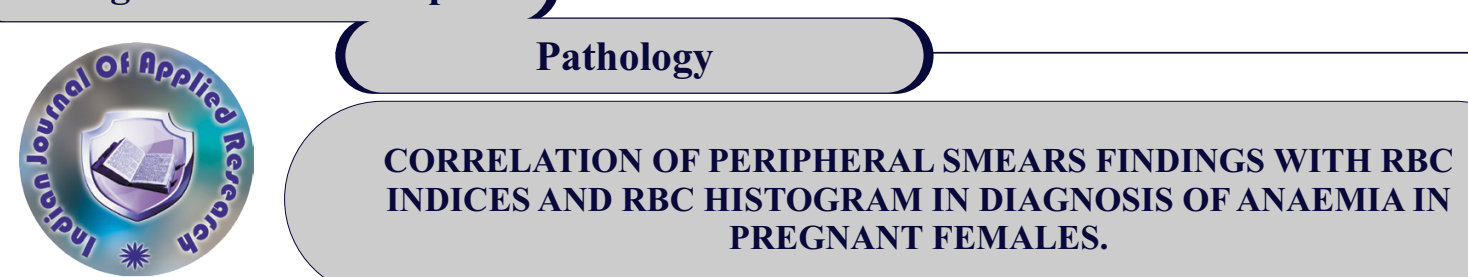

\begin{tabular}{ll}
\hline Dr. Preethi. M* & $\begin{array}{l}\text { Third year Post Graduate student, Navodaya Medical College, Raichur. } \\
\text { *Corresponding Author }\end{array}$ \\
\hline Dr. Ashraf A.Z & Assistant Professor, Navodaya Medical College, Raichur. \\
\hline Dr. A.S. Anand & Professor \& Head; Department of Pathology, Navodaya Medical College, Raichur. \\
\hline Dr. Yaranal P.J & Professor, Navodaya Medical College, Raichur. \\
\hline
\end{tabular}

ABSTRACT) BACKGROUND: Red Blood Cell (RBC) histogram is a graphic representation of particle size distribution (cell frequencies verses size). RBC histogram with complete blood cell counts by automated analyser provides an idea about morphological changes of red blood cells in anaemias. Peripheral smear examination (PBS) helps in diagnosing different types of anaemias by visualizing abnormal shape and size, arrangement and immature red cells. This study was intended to analyze various types of anemia in pregnant women and to correlate RBC indices, $\mathrm{RBC}$ histogram with peripheral smear.

\title{
AIMS AND OBJECTIVES:
}

1) To Correlate RBC indices, RBC histogram with peripheral smear in anemic pregnant women.

2) To categorize the type of anemia based on RBC indices, histogram and peripheral smear.

MATERIALS AND METHODS: Venous blood sample was run in SYSMEX XNL/350 six-part analyser. The haematological investigations of $\mathrm{CBC}$, red blood cell indices (MCV, MCH, MCHC) and RBC histogram were obtained by analyser. Peripheral blood smear study was performed. RESULTS: The present study included 55 cases among which in decreasing order of frequency, NNA (50.9\%) was more common followed by MHA (40\%), macrocytic anaemia (3.63\%) and dimorphic anaemia $(5.45 \%)$, as categorized by peripheral smear examination. Analysis by erythrocyte indices and histogram showed similar findings except in normocytic, microcytic and dimorphic which were $13.63 \%, 32.14 \%$ and $1.8 \%$ respectively. The correlation was done between the diagnosis made by the two methods which showed $76 \%$ concordance and discordance of $23.63 \%$. CONCLUSION: Findings of the present study shows automated analyzer correlated well with PBS in cases of macrocytic and dimorphic anemia, compared to normocytic normochromic and microcytic hypochromic anaemia. As, RBC histogram with RBC indices and PBS are supplementary to each other, both should be used in conjunction for accurate diagnosis.

\section{KEYWORDS : Peripheral Blood Smear, RBC Indices, RBC Histogram, Anaemia And Pregnancy.}

\section{INTRODUCTION}

Anaemia is a decrease in the oxygen carrying capacity of the blood. It can arise if the hemoglobin $(\mathrm{Hb})$ concentration of the red blood cells (RBCs) or the packed cell volume of RBCs (PCV) is below the lower limit of the reference interval for the individual's age, gender, geographical location, and physiological status.

During pregnancy the total blood volume increases by about 1.5 liter. The plasma volume increases more compared to red cell mass which leading to hemodilution and reduced hemoglobin concentration. This is termed physiological anemia of pregnancy. The World Health Organization (WHO) has suggested that anemia is present in pregnancy when $\mathrm{Hb}$ level is $<11 \mathrm{~g} / \mathrm{dl}^{3}$

Until recently, hematological tests were performed only by manual methods. These methods, though still performed in many peripheral laboratories, are labor-intensive and involve use of hemocytometers, centrifuges, wintrobe tubes, photometers and stained blood smears. Hematology cell analysers can generate the blood test results rapidly and also perform additional tests not possible by manual technology.

The automated hematolyzers are based on impedance principle and relies on the change in conductance as each cell passes through an aperture. This change in conductance results in development of an electrical pulse the amplitude of which is proportional to the cell volume. The results are displayed as numericals and histogram. ${ }^{1} \mathrm{RBC}$ histogram is a standard part and routinely generated by automated cell analyzers. ${ }^{5,6}$ The well known Coulter principle of counting and sizing the cells provides basis of generating a histogram.

Histograms are graphical representation of cell frequencies verses size. $\mathrm{X}$ axis represents the size of cell and $\mathrm{Y}$ axis represents number of cells. ${ }^{3}$ Various shapes of histogram give hint about the pathology before the blood smear could be examined. Histogram is helpful in comparing the size of patient's cell with normal population. ${ }^{1}$ Shift in one direction or another can be of diagnostic importance and in association with other CBC parameters such as RBC distribution width (RDW) and $\mathrm{RBC}$ indices (MCV, MCH, MCHC) it has been found abnormal in various hematological conditions.

RBC Indices were first introduced by Wintrobe to define the size and hemoglobin content. Normal values of MCV is considered $80-100 \mathrm{fl}$, $\mathrm{MCH}$ between 27-32 pg and MCHC between $30-35 \mathrm{~g} / \mathrm{dl}{ }^{4}$ The normal RDW-CV is $11.5 \%$ to $14.5 \%$ and normal RDW-SD is 39 to $47 \mathrm{fL}$. Increased RDW shows a mild broad base histogram. Normal red cell histograms are symmetric, single peaked, and Guassian or "bell shaped"curve. The distribution should always start and end on base line and should be located between the two discriminators, majority of each cell falls between $55 \mathrm{fL}$ and $125 \mathrm{fL}$.

When the distribution is not symmetric, it is referred to as skewed.

1. Left skewed: If the cells are smaller than normal, the curve will be more to the left as in untreated iron deficiency anemia.?

2. Right skewed: If the cells are larger than normal, the histogram curve will be more to right as in megaloblastic anemia.

3. Broad Base: The population of cells would be variable in size. The broad base would represent the RDW above the reference range.

4. Bimodal: Two distinct populations of RBC's are present with increased RDW and histogram may have more than one peak. ${ }^{4}$

5. Short Peak: maximum populations of cells are of same size and histogram shows narrow curve. ${ }^{4}$

Histogram becomes a practical tool in initial stage of morphological analysis if combined with knowledge of $\mathrm{CBC}$ parameters such as red cell indices and RDW. To make the histogram more meaningful it should be compared with a reference normal curve or confirmed by microscopy.

Since decades, peripheral blood smear has been used as window to observe hematological ongoings. Analyzing peripheral blood smears routinely has facilitated interpretation of various hematological disorders and has been a major diagnostic tool. Cell counters have penetrated medical laboratory services in a ubiquitous manner with increasing efficacy and decreasing cost all over the world. Over the past few years complete blood count (CBC) by the automated haematology analysers and microscopic examination of peripheral smear, have complemented each other to provide a comprehensive report on patient's blood sample.

In this present study, correlation of peripheral smear with RBC indices and RBC histogram obtained from automated hematology analyser of 
anemic pregnant women was done and various types of anemia were categorised.

\section{MATERIALS AND METHODS}

The present study was a prospective and observational study performed at Navodaya Medical College Hospital and Research Centre, Raichur, over a period of six months between November 2019 to May 2020 which included anemic pregnant women between 18- 39 years with $\mathrm{Hb}<11 \mathrm{~g} \%$. A detailed history was obtained and those who had undergone medical treatment for anemia and blood transfusion in last three months were excluded.

Venous blood was collected under aseptic precautions in EDTA vaccutainer for hematological investigations. The investigations were performed on SYSMEX XNL/350 six-part haematology analyser with standard calibration. $\mathrm{CBC}, \mathrm{RBC}$ indices, RDW and $\mathrm{RBC}$ histogram were obtained by analyzer. A good peripheral smear was done and stained with Leishman's stain following standard operating procedure and morphological abnormalities of red blood cells were observed.

Typing of anemia based on RBC indices, RDW and histogram was categorized based on standard references ${ }^{(4,7)}$ as following

- $\quad$ Left skewed

- Right skewed

- Broadbase

- Bimodal

- Short peak

And morphological typing of anemia on PBS examination was categorized as

- Microcytic Hypochromic anaemia

- Normocytic Normochromic anaemia

- Macrocytic anaemia

- Dimorphic anaemia

The findings obtained by PBS microscopic examination was correlated with histograms, RBC indices and RDW.

Concordance was considered when classification of anemia based on PBS correlated with classification of anemia based on histogram, RBC indices and RDW i.e when

Normocytic Normochromic Anaemia showing normal curve i.e bell shaped curve and peak between 80-110fl.

Microcytic Hypochromic anaemia showing left skew with peak of the curve before $80 \mathrm{fl}$

Macrocytic Anaemia showing right skew with peak of the curve beyond $110 \mathrm{fl}$.

Dimorphic Anaemia showing curve with two peaks.

\section{RESULTS}

A total number of 55 patients included in the study and were categorized from 18-39 years age groups, at interval of 5 years.

Majority of cases (33 cases, 60\%) were found in the age group of 18-25 years. (Table 1)

Table 1: Age distribution of patients studied

\begin{tabular}{|c|c|c|}
\hline Age in years & Number of patients $(\mathbf{n}=\mathbf{5 5})$ & Percentage (\%) \\
\hline $18-25$ & 33 & 60 \\
\hline $26-30$ & 15 & 27.27 \\
\hline $31-35$ & 7 & 12.72 \\
\hline $36-39$ & 0 & 0 \\
\hline
\end{tabular}

On PBS examination maximum number of cases showed features of normocytic normochromic anaemia and least number of cases belonged to macrocytic anaemia.(Table 2)

Table 2: Categorization of anaemia based on Peripheral smear

\begin{tabular}{|c|c|c|}
\hline $\begin{array}{c}\text { Peripheral smear } \\
\text { Examination }\end{array}$ & $\begin{array}{c}\text { Number of } \\
\text { patients }(\mathbf{n}=\mathbf{5 5})\end{array}$ & Percentage (\%) \\
\hline Normocytic Anaemia & 28 & 50.90 \\
\hline $\begin{array}{c}\text { Microcytic hypochromic } \\
\text { Anaemia }\end{array}$ & 22 & 40 \\
\hline Macrocytic Anaemia & 2 & 3.63 \\
\hline Dimorphic Anemia & 3 & 5.45 \\
\hline
\end{tabular}

Normal curve on histogram was considered when cell size was between $55 \mathrm{fl}-125 \mathrm{fl}$ and peak was noted between $80-110 \mathrm{fl}$.
Left skewed: peak of the curve is seen before $80 \mathrm{fl}$.

Right skewed: peak of the curve is noted beyond $110 \mathrm{fl}$

Broad Base: curve is seen starting before $55 \mathrm{fl}$ and ends after $100 \mathrm{fl}$.

Bimodal: two peaks (camel humps) is seen.

Short Peak: the curve starts and ends within an interval of $30-40 \mathrm{fl}$.

Table 3: Categorization based on histogram findings

\begin{tabular}{|c|c|c|}
\hline Types of Histogram & No of patients (n= 55) & Percentage (\%) \\
\hline Normal Curve & 21 & 38.18 \\
\hline Left shift & 16 & 29.09 \\
\hline Right shift & 2 & 3.63 \\
\hline Broad Base & 11 & 20 \\
\hline Bimodal & 3 & 5.45 \\
\hline Short Peak & 2 & 3.63 \\
\hline
\end{tabular}

In present study, normocytic normochromic anaemia cases showed $\mathrm{MCV}, \mathrm{MCH}, \mathrm{MCHC}$ and RDW within normal limits including normal curve, with $23.6 \%$ cases showing mild increase in RDW representing broad base. In microcytic hypochromic anemia cases $\mathrm{MCV}, \mathrm{MCH}$ were less than normal range, with normal MCHC showing left sided skewed curve and increased RDW due to anisopoikilocytosis, showing broad base. In Macrocytic anemia there was increase in $\mathrm{MCV}, \mathrm{MCH}$, RDW with normal MCHC. Due to variation in size \& shape of the RBCs, curve showed right skewing with increased RDW and broad base. In Dimorphic anemia, $\mathrm{MCV}, \mathrm{MCH}, \mathrm{MCHC}$ were in the normal limits and RDW was increased due to high degree of anisopoikilocytosis, showing left or right skewing with broad base.(Table 4)

Table 4: Comparison of PBS with histogram and RBC indices

\begin{tabular}{|l|c|c|c|c|c|c|}
\hline & $\begin{array}{c}\text { Normal } \\
\text { curve }\end{array}$ & Left shift & Right shift & $\begin{array}{c}\text { Broad } \\
\text { base }\end{array}$ & $\begin{array}{c}\text { Short } \\
\text { Peak }\end{array}$ & Bimodal \\
\hline Normocytic & 19 & & & 9 & & \\
\hline Microcytic & & 17 & & 3 & 2 & \\
\hline Macrocytic & & & 2 & & & \\
\hline Dimorphic & & & & 1 & & 2 \\
\hline
\end{tabular}

Concordance was considered when classification of anaemia based on PBS correlated with classification of anaemia based on histogram, $\mathrm{RBC}$ indices and RDW.

Table 5: Correlation between Automated and Manual Morphological typing (PBS) of Anaemia

\begin{tabular}{|c|c|c|}
\hline & Concordant & Discordant \\
\hline Normocytic normochromic & 19 & 9 \\
\hline Microcytic hypochromic & 19 & 3 \\
\hline Macrocytic & 2 & 0 \\
\hline Dimorphic & 2 & 1 \\
\hline Total & 42 & 13 \\
\hline
\end{tabular}

Majority of cases of macrocytic anaemia were concordant whereas significant numbers of cases ( 9 cases) of normocytic normochromic anaemia were discordant.

\section{DISCUSSION}

In present study the age range of the population was between 18 to 39 years and maximum cases were noted in the age group 18- 25 years. Predominant cases were in young age group and similar finding was observed by Sandhya et al in their study.

When RBC population is homogenous it gives narrow distribution curve and a broad base curve which usually denotes presence of significant anisocytosis, and can be confirmed by PBS examination. Shift of histogram depends on the size of RBC, If cell size is more than normal (macrocytic RBC), shift is towards right and when size of cell is less than normal (microcytic RBC) shift is towards left.

In the present study out of 55 cases, 21 showed normal curve, 16 left shift, 2 right shift, 12 broad base, 2 short peak and 2 were of bimodal.

Among 28 cases $(50.90 \%)$ of normocytic normochromic anaemia, 19 cases $(67.85 \%)$ showed normal curve, rest 9 cases $(32.14 \%)$ were of broad base category. In normocytic normochromic anemia, the red cell indices like $\mathrm{MCV}, \mathrm{MCH}$ and $\mathrm{MCHC}$ were within the normal limits, with $39.14 \%$ cases showing mild increase indices with broad base in most of the histograms. On peripheral smear examination there was predominance of normocytes along with microcytes, which resulted in higher deviation and hence the RDW was high. These findings correlated with study carried out by Chavda et al. ${ }^{1 /}$ 
Out of 22 cases $(40 \%)$ of microcytic hypochromic anemia, 17 cases (77.27\%) showed left shift, 2 cases $(9.09 \%)$ showed short peak, 3 cases (13.63\%) showed broad base. In the cases of microcytic hypochromic anaemia, $\mathrm{MCHC}$ was normal but MCV and $\mathrm{MCH}$ were decreased. Red blood cell population with low MCV will be skewed towards left. A broad base curve because of high RDW represents anisocytosis. RDW is also increased when there is increased number of smaller cells. These findings correlated with study carried out by Sandhya et al and Chavda et al. ${ }^{10,11}$

In present study macrocytic anaemia due to variation in size and shape of the RBC's, increased MCV, RDW, MCH were noted with normal MCHC. Both cases $(3.63 \%)$ of macrocytic anemia showed right skewed curve. This finding correlated with study done by Sandhya et al. ${ }^{10}$ Right shift curve correlated well with increased MCV and $\mathrm{MCH}$.

Out of 3 cases (5.45\%) of dimorphic anemia, 2 cases (66.6\%) showed bimodal peak, one case $(33.33 \%)$ showed broad base. In cases of dimorphic anemia, on peripheral smear examination dual population of microcytic \& normocytic or normocytic \& macrocytic red cells or admixture of different sized RBC's were observed hence the histogram has two distinct peaks. In present study, in dimorphic anaemia cases $\mathrm{MCV}, \mathrm{MCH}$ and $\mathrm{MCHC}$ were normal and RDW was increased due to marked anisocytosis. These findings correlated with study carried out by Sandhya et al and Chavda et al. . $^{10,11}$

Table 6: Comparison of histogram findings of different studies

\begin{tabular}{|l|l|l|l|}
\hline Histograms & Sandhya et al. $^{10}$ & Chavda J et al. $^{11}$ & Present Study \\
\hline Normal Curve & $13 \%$ & $19 \%$ & $34.54 \%$ \\
\hline Left Skew & $30 \%$ & $27 \%$ & $30.90 \%$ \\
\hline Right Skew & $06 \%$ & $07 \%$ & $3.63 \%$ \\
\hline Broad Base & $40 \%$ & $38 \%$ & $23.63 \%$ \\
\hline Bimodal & $04 \%$ & $03 \%$ & $3.63 \%$ \\
\hline Short Peak & $05 \%$ & $06 \%$ & $3.63 \%$ \\
\hline
\end{tabular}
In the present study 3 of 22 cases (13.63\%) of microcytic hypochromic anaemia had high RDW which is normally expected, but 9 of 28 cases (32.14\%) of normocytic normochromic anaemia showed raised RDW. This discrepancy may result in misclassification for the diagnosis of anaemia causing discordance. However, the mean RDW value among the cases of microcytic hypochromic anaemia with raised RDW was $17.7 \%$, but the mean RDW in cases with normocytic normochromic anaemia was $14.1 \%$. In such cases peripheral smear examination helps to identify the morphology of cells.

In present study results of histogram and peripheral smear were varied. Concordant typing was present among 42 cases and discordance was present among 13 cases. Discordance can be due to presence of agglutinated RBCs, fragmented RBCs or abnormal blood cells, not detected by automated analyzer and later was corrected by smear examination.(Table 5)

Table 7: Comparison of concordant and discordant cases in different studies

\begin{tabular}{|c|c|c|c|}
\hline & Total & Concordant & Discordant \\
\hline Farah E. et al, 201312 & 350 & $274(78.3 \%)$ & $76(21.7 \%)$ \\
\hline Radadiya P et al, 201513 & 100 & $72(72 \%)$ & $28(28 \%)$ \\
\hline Present study & 55 & $42(76.36 \%)$ & $13(23.63 \%)$ \\
\hline
\end{tabular}

Aslina et al found that peripheral blood smear was more sensitive than $\mathrm{RBC}$ indices for identifying early microcytic changes because the $\mathrm{MCV}$ represented the mean of the distribution curve and was insensitive to the presence of small numbers of macrocytes, ${ }^{14}$ this finding was similar in the study conducted by Lantis et al and the present study. ${ }^{15}$

Peripheral blood smears can differentiate not only morphological types of anaemia, but also hemoglobinopathies and other blood disorders. The validation technique of automated hematology analyzer with peripheral smear should be implemented in each lab especially to assure performance of test for the maximum benefit of patients.

\section{CONCLUSION}

Study concluded that by both autoanalyzer and PBS examination, NNA was the commonest anaemia in pregnant females. Findings of automated analyzer correlated well with PBS in cases of macrocytic and dimorphic anaemia, compared to normocytic normochromic and microcytic hypochromic anaemia. The discrepancy between automation and manual scan of peripheral blood in the analysing red blood cell morphology can result in misclassification of anaemia. As the RBC parameters obtained from autoanalyser and PBS examination are supplementary to each other, both should be used in conjunction for accurate haematological diagnosis.

\section{REFERENCES}

1. Turgeon, M.L. Clinical Hematology: Theory and Procedures. Wilkins, Lippincott Williams, 5th ed, Philadelphia; 2012 .

2. Rodak B, Fritsma G, Keohane E. Hematology: Clinical Principles and Applications. 4th ed. Elsevier: Saunders; 2012.

3. Chandra S, Tripathi A K, Mishra S, Amzarul M, Vaish A K. Physiological changes in hematological parameters during pregnancy. Indian Journal of Hematology and Blood Transfusion.2012;28(3):144-146.

4. Kawthalkar S. Essentials of Clinical Pathology. Automation in hematology. Jaypee; 2012;(1):319-326.

5. Bessman J D, Gilmer P R, Gardner F H. Improved classification of anemias by MCV and RDW. Am J Clin Pathol. 1983;80:322-326

6. Williams L J. Cell histograms: New trends in data interpretation and classification. J Med Technol.1984;3:189-197.

7. Shifa Maqsood, Sharadrutha A. Study of RBC histograms in various anemias: A six months prospective study. Perspectives in Medical Research. 2019; 7(1):40-44.

8. Constantino BT. The red cell histogram and the dimorphic red cell population. LabMed. 2011;42:300-308

9. Shruti S, Sanjay B, Kusum J. Comparative study of anaemia cases based on peripheral blood smears and cell counter generated red cell indices. Medpulse - international medical journal. 2017;4(1):44-48

10. Sandhya I, Muhasin T P. Study of RBC Histogram in Various Anemias. Journal of Evolution of Medical and Dental Sciences. 2014;3(74):15521-15534.

11. Chavda J, Goswami P, Goswami A. RBC histogram as diagnostic tool in anemias. IOSR Journal of Dental and Medical Sciences. 2015; 14(10):19-22.

12. Farah E, Mehwish A, Nafisa H. Comparative Study in the Diagnosis of Anemia by Sysmex Kx-21n Hematology Analyzer with Peripheral Blood Smear. International Journal of Endorsing Health Science Research (IJEHSR). 2013;1(2):89.

13. Radadiya P, Mehta N, Goswami H. Automated red blood cell analysis compared with routine red blood cell morphology by smear review. NHL Journal of Medical Sciences. 2015;4(1):53-57.

14. Aslinia F, Mazza J J, Yale S H. Megaloblastic anemia and other causes of macrocytosis. Clin Med Res

2006;4:236-41

15. Lantis K, Harris R, Davis G, Renner N, Finn W. Elimination of instrument - driven reflex manual differential leukocyte counts optimization of manual blood smear review criteria in a high volume automated hematology laboratory. American Journal of Clinical Pathology. 2003;119(5):656-662 